\title{
Rifampin combination therapy in staphylococcal prosthetic joint infections: a randomized controlled trial
}

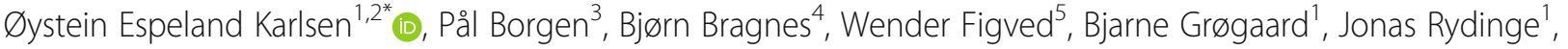 \\ Lars Sandberg ${ }^{6}$, Finnur Snorrason ${ }^{1}$, Helge Wangen ${ }^{7}$, Eivind Witsøe ${ }^{8}$ and Marianne Westberg ${ }^{1}$
}

\begin{abstract}
Background: The evidence supporting rifampin combination therapy in prosthetic joint infections (PJI) is limited due to the lack of controlled studies. The aim of this study is to evaluate the effect of adding rifampin to conventional antimicrobial therapy in early staphylococcal PJls treated with debridement and retention of the implant (DAIR).
\end{abstract}

Methods: In this multicenter randomized controlled trial, 99 patients with PJI after hip and knee arthroplasties were enrolled. They were randomly assigned to receive rifampin or not in addition to standard antimicrobial treatment with cloxacillin or vancomycin in case of methicillin resistance. The primary endpoint was no signs of infection after 2 years of follow-up.

Results: Forty-eight patients were included in the final analyses. There were no differences in patient characteristics or comorbidities between the two groups. There was no significant difference in remission rate between the rifampin combination group (17 of $23(74 \%)$ ) and the monotherapy group (18 of 25 (72\%), relative risk 1.03, 95\% confidence interval 0.73 to $1.45, p=0.88)$.

Conclusion: This trial has not proven a statistically significant advantage by adding rifampin to standard antibiotic treatment in acute staphylococcal PJls.

Trial registration: The Regional Ethics Committee and the Norwegian Medicines Agency approved the study (EudraCT 2005-005494-29), and the study was registered at ClinicalTrials.gov at Jan 18, 2007 (NCT00423982).

Keywords: Rifampin, Staphylococci, Prosthetic joint infection, Surgery

\section{Highlights/summary}

Rifampin is increasingly used in staphylococcal prosthetic joint infections treated with debridement and retention of the prosthesis. This study is the largest randomized controlled study on this subject. No

\footnotetext{
* Correspondence: oekarl00@hotmail.com

1 Division of Orthopaedic Surgery, Oslo University Hospital, Oslo, Norway ${ }^{2}$ Department of Orthopaedic Surgery, Betanien Hospital, Skien, Norway

Full list of author information is available at the end of the article
}

statistical significant advantage by adding rifampin to the antimicrobial medication is shown.

\section{Introduction}

The number of patients requiring prosthetic joint replacement is increasing due to good functional outcome and excellent pain relief in a growing population of the elderly [1]. Prosthetic joint infection (PJI) is a rare but devastating complication occurring in $1-2 \%$ of primary interventions [2,3] and in $2-20 \%$ of revision procedures [4]. PJI leads to increased morbidity, long periods of

C The Author(s). 2020 Open Access This article is licensed under a Creative Commons Attribution 4.0 International License, which permits use, sharing, adaptation, distribution and reproduction in any medium or format, as long as you give appropriate credit to the original author(s) and the source, provide a link to the Creative Commons licence, and indicate if changes were made. The images or other third party material in this article are included in the article's Creative Commons licence, unless indicated otherwise in a credit line to the material. If material is not included in the article's Creative Commons licence and your intended use is not permitted by statutory regulation or exceeds the permitted use, you will need to obtain permission directly from the copyright holder. To view a copy of this licence, visit http://creativecommons.org/licenses/by/4.0/ The Creative Commons Public Domain Dedication waiver (http://creativecommons.org/publicdomain/zero/1.0/) applies to the data made available in this article, unless otherwise stated in a credit line to the data. 
hospitalization, and high costs [3,5-7]. Of concern, the absolute number of PJI is increasing due to the increasing number of joint replacements. Also, the risk of infection seems to be increasing in recent years $[8,9]$.

Coagulase-negative staphylococci (CoNS) and Staphylococcus aureus are the most frequent cause of PJI, accounting for $30-47 \%$ and $12-44 \%$, respectively [ 10 14]. Staphylococci are biofilm-forming bacteria [15]. The microbes adhere to prostheses and adjacent tissues and are enclosed in a polymeric matrix, where they are protected from the host immune response and antimicrobials. This makes the eradication of PJI difficult [16-18].

In acute PJI and acute hematogenous spread PJI, debridement and implant retention (DAIR) combined with antimicrobial treatment is an attractive surgical option due to its lesser surgical trauma and hence limited functional impairment, but the results vary greatly in the literature $[13,19,20]$. Rifampin is a broad-spectrum antimicrobial agent that is a frontline drug in the treatment of tuberculosis, but also acts bactericidal against $S$. aureus. It penetrates the biofilm and is able to kill sessile bacteria [21]. Due to the rapid development of resistance, rifampin must never be used alone, but in combination with another antimicrobial agent. Further, there are challenges with drug interactions, reported in up to $52 \%$ of patients treated with rifampin-combination therapy for infective endocarditis [22].

Rifampin appears to be promising in treating serious gram-positive implant-related infections [16, 23-25]. However, evidence to support the adjunctive use of rifampin in PJI-treatment is week and based on one small, randomized controlled trial and some observational studies [23-28]. Due to the absence of randomized controlled trials, and the limitations of the existing literature, there is still a debate regarding the role of rifampin in staphylococcal PJIs.

The aim of this trial was to evaluate the effect of adding rifampin to a standard antimicrobial therapy with cloxacillin or vancomycin in early postoperative and acute hematogenous staphylococcal PJI.

\section{Patients and methods}

\section{Study design and participants}

This open-label, randomized controlled trial was conducted in five district general hospitals, one specialized orthopedic hospital and two university hospitals in Norway. Recruitment was from January 2006 to January 2012, with a final follow-up scheduled at 2 years. Eligible patients were adult men and women operated with a total hip or knee prosthesis, with clinical signs suggesting early postoperative or acute hematogenous PJI and with a stable implant in place. Confirmed infections due to $S$. aureus or CoNS were included in the study. Positive cultures in the expected aseptic revision were not included. Exclusion criteria were PJI with other bacteria than staphylococci, less than 2 years expected survival, inability to comply with treatment and/or follow-up visits, and contraindications to the use of rifampin, cloxacillin, or vancomycin. The Regional Ethics Committee and the Norwegian Medicines Agency approved the study (EudraCT 2005-005494-29), and the study was registered at ClinicalTrials.gov (NCT00423982). Written informed consent was obtained from all patients before inclusion. The study has been performed according to the principles of the Declaration of Helsinki.

\section{Definition of PJ}

PJI was suspected when patients presented with pain, redness, or wound discharge within 30 days after prosthetic surgery (acute postoperative PJI) or with an acute hematogenous PJI with symptoms for less than 3 weeks [13]. During the DAIR procedure, eight intraoperative tissue specimens were collected with separate instruments, of which one from periprosthetic bone and one from synovial fluid. At least two of the specimens had to be positive with the same microbe to define PJI.

\section{Randomization}

Patients were randomized at admission to hospital to conventional antimicrobial therapy with or without the adjunction of rifampin. Randomization was stratified by center and performed by a randomization generator by blocks of 10 .

\section{Surgical treatment}

All included patients underwent a highly standardized soft tissue revision, with thorough debridement including excision of the wound. New instruments were introduced after suprafascial incision. The implants were left in place, but modular components were exchanged. The wound was cleansed with pulsatile irrigation with $9 \mathrm{~L}$ of saline. After the DAIR procedure, new draping and instruments were introduced, including the new modular components. Two $10 \times 10 \mathrm{~cm}$ gentamicin-containing collagen sponges, each containing $130 \mathrm{mg}$ gentamicin sulphate, were placed in the wound before closure. Finally, the wound was sutured in layers. No drains were used.

\section{Antimicrobial therapy}

The first dose of antibiotics was given perioperatively immediately after the 8 tissue specimens were collected. All patients were given cloxacillin $2 \mathrm{~g} \times 4$ and vancomycin $1 \mathrm{~g} \times 2$ intravenously until microbiological results were known. Patients randomized to the rifampincombination group were in addition treated with oral rifampin from day 1 after surgery. When cultures proved methicillin-susceptible $S$. aureus or CoNS, rifampin 300 
$\mathrm{mg} \times 3$ orally and cloxacillin $2 \mathrm{~g} \times 4$ intravenously were given for 2 weeks, then rifampin $300 \mathrm{mg} \times 3$ orally and cloxacillin $1 \mathrm{~g} \times 4$ orally for 4 weeks. In case of methicillin-resistant Staphylococcus epidermidis (MRSE), patients were treated with rifampin $300 \mathrm{mg} \times 3$ orally and vancomycin $1 \mathrm{~g} \times 2$ intravenously for 6 weeks. In the monotherapy group, when proven methicillinsusceptible staphylococci, cloxacillin $2 \mathrm{~g} \times 4$ intravenously was given for 2 weeks, then cloxacillin $1 \mathrm{~g} \times 4$ orally for 4 weeks. In case of MRSE, patients were treated with vancomycin $1 \mathrm{~g} \times 2$ intravenously for 6 weeks.

Vancomycin serum levels were monitored 2 times per week, and the vancomycin dose was adjusted if the serum level outranged the recommended plasma concentration levels.

\section{Follow-up}

Medical conditions and medications prior to surgery were recorded, as well as demographic data. Patients were clinically assessed at enrolment, during the hospital stay, and regularly throughout the treatment period. Hematological status, serum-creatinine, and hepatic enzymes were analyzed before treatment and during antimicrobial treatment to determine any toxic side effects. C-reactive protein (CRP) and erythrocyte sedimentation rate (ESR) were used to assess treatment effectiveness.

The cure was defined as the lack of clinical signs and symptoms of PJI (fever, joint pain, erythema, warmth of the skin around the incision, and sinus tract), CRP $<10$ $\mathrm{mg} / \mathrm{ml}$, ESR as prior to index operation, and no radiological signs of loosening at 2 years of follow-up.

Confirmed failure was defined as re-revision with the isolation of the initial or other microorganisms from a minimum of two intraoperative tissue specimens during the 2-year study period. Probably, failure was defined if clinical signs and symptoms of local infection but without microbiological documentation. Both groups were considered failures in the analysis. Repeated DAIR procedures were considered being a failure.

\section{Statistical analysis}

Based on data from in-hospital quality registers, we assumed a cure rate of $70 \%$ following a DAIR procedure without the addition of rifampin. An increase in the cure rate of $20 \%$ may be proven with a statistical power of $80 \%$ when including 62 patients in each group. Taking into account the expected dropouts, we intended to include 100 patients in each group.

Analyses were conducted according to a modified intention-to-treat principle. Time to failure was estimated with the Kaplan-Meier method, and the log-rank test was used to compare groups. The chi-square or Fisher's exact test was used to compare categorical and continuous variables in the rifampin group and the monotherapy group. A $P$ value $<0.05$ was considered significant. We used SPSS for Windows, version 23 (SPSS inc, Chicago, IL, USA) for the analyses.

\section{Results}

Overall, 99 patients with suspected PJI were enrolled in the study, of which 65 had a proven staphylococcal infection. The last follow-up visit was in January 2014. Recruitment for the trial was slower than anticipated, and the study was stopped before reaching the estimated sample size. In addition, an increasing trend towards using rifampin developed in the orthopedic society during the study period, which also made inclusion more difficult. Figure 1 displays the study profile.

Forty-eight patients were included in the final analyses, 23 in the rifampin-combination group, and 25 in the monotherapy group. Baseline characteristics were similar between the groups (Table 1). The median age was 68.5 years (range 37-92). S. aureus was found in 36 of the participants and CoNS in 14. Two patients had a combination of $S$. aureus and methicillin-susceptible $S$. epidermidis. Further, there was one infection with Staphylococcus lugdunensis and one Staphylococcus capitis, both susceptible to methicillin (Table 2).

The median follow-up was 27 months (range 18-99) in the rifampin-combination group and 27 months (range 7-106) in the monotherapy group. Some of the latest follow-ups were conducted as telephone interviews. The two patients with the shortest follow-up were deceased before final the follow-up, but were reported to be infection-free at the time of death.

\section{Outcome at 2 years}

There was no statistically significant difference between the two treatment groups in the success of DAIR procedure in the eradication of acute staphylococcal PJI; the success rate at 2 years was $17 / 23(74 \%)$ in the rifampincombination group and 18/25 (72\%) in the monotherapy group (95\% CI $0.73-1.45 ; p=0.88$ ). A successful DAIR procedure is of important clinical relevance for the patients, as further revision arthroplasty, and thereby possible functional impairment, is not needed. A Kaplan-Meier plot is used to show time to failure in the two groups (Fig. 2).

Subgroup analyses according to the type of staphylococci showed a cure rate for S. aureus infections of 14/ 18 in the rifampin group and 13/20 in the monotherapy group (95\% CI $0.80-1,80 ; p=0.49$ ). CoNS infections had a cure rate of $5 / 5$ in the monotherapy group and $3 /$ 5 in the rifampin-combination group (95\% CI 0.29-1.22; $p=0.44$ ) (Table 3). These two groups were also similar in age, sex, and comorbidities. 


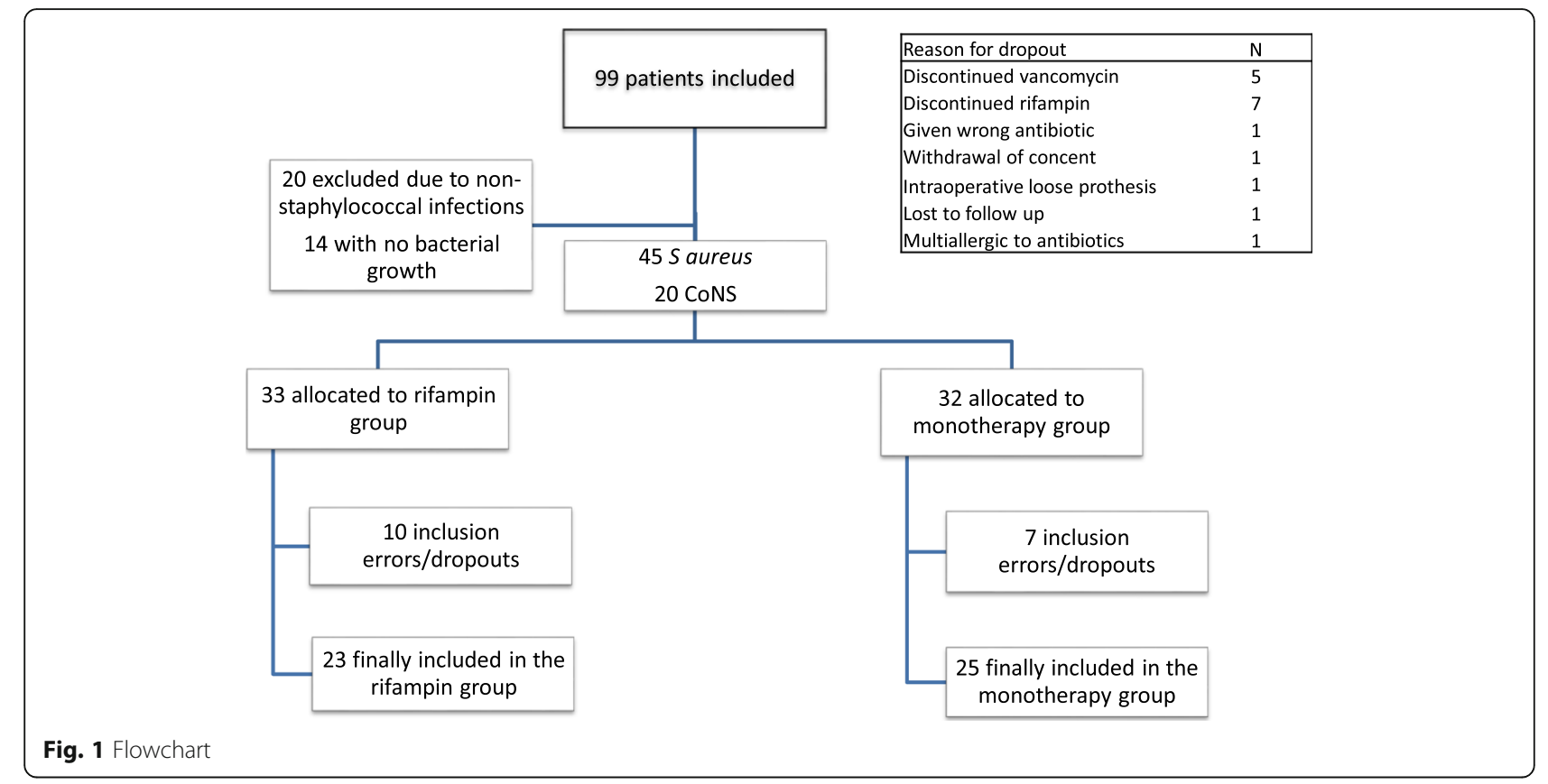

Table 1 Baseline characteristics of the 48 patients

\begin{tabular}{|c|c|c|c|}
\hline Characteristics & Rifampin group $(n=23)$ & Monotherapy group $(n=25)$ & Total $(n=48)$ \\
\hline Age, year, median (range) & $70(37-92)$ & $66(39-84)$ & $68.5(37-92)$ \\
\hline Sex, male (\%) & $15(65)$ & $17(68)$ & $32(67)$ \\
\hline ASA scores $1-2$, no $(\%)$ & $16(70)$ & $21(84)$ & $37(77)$ \\
\hline BMl, mean (SD) & $30.1(1.3)$ & $27(1.0)$ & $28.4(0.8)$ \\
\hline Diabetes mellitus & 3 & 3 & 6 \\
\hline Immunosuppressive medication & 2 & 2 & 4 \\
\hline Smoking & 3 & 4 & 7 \\
\hline Time from index surgery to revision, median, days (range) & $19(7-912)$ & $17(8-122)$ & $18(7-912)$ \\
\hline \multicolumn{4}{|l|}{ Hip prosthesis } \\
\hline Primary hip prosthesis & 17 & 14 & 31 \\
\hline Revision hip prostehesis & 3 & 5 & 8 \\
\hline \multicolumn{4}{|l|}{ Knee prosthesis } \\
\hline Primary knee prosthesis & 3 & 6 & 9 \\
\hline CRP pre surgery, mean (SD) & $135(21.1)$ & $167(26.4)$ & $151(16.9)$ \\
\hline Creatinin pre surgery, mean (SD) & $78(5.7)$ & $79(4.4)$ & $79(3.5)$ \\
\hline \multicolumn{4}{|l|}{ Type of prosthesis ${ }^{a}$} \\
\hline Cemented prosthesis & 14 & 16 & 30 \\
\hline Non cemented & 4 & 5 & 9 \\
\hline Reverse hybrid & 4 & 4 & 8 \\
\hline
\end{tabular}

ASA American Society of Anesthesiologists physical status classifications system, $B M I$ body mass index ${ }^{\text {a Missing data, } n=1}$ 
Table 2 Bacterial findings in initial DAIR procedure

\begin{tabular}{llll}
\hline Microbes & Rifampin-combination group & Monotherapy group & Total \\
\hline MSSA & 15 & 19 & 34 \\
MRSE & 5 & 5 & 10 \\
MSSA + MSSE & 2 & 0 & 2 \\
Staph lugdunensis & 1 & 0 & 1 \\
Staph capitis & 0 & 1 & 1 \\
\hline
\end{tabular}

MSSA methicillin-susceptible S. aureus, MRSE methicillin-resistant S. epidermidis

The bacteria found in the revisions after failures are listed in Table 4. There was no development of rifampin resistance in the two patients from the rifampincombination group with positive cultures.

\section{Adverse events}

Of the 65 patients initially included in the study, only 4 of 31 who were assigned to rifampin treatment dropped out or were excluded due to side effects or discontinuation of rifampin. One patient using rifampin was reported with hepatic failure. For vancomycin, 5 patients dropped because of side effects, but as many as 13 patients developed increased serum-creatinine levels that led to the discontinuation of vancomycin. Eight out of these 13 continued in the study because their bacteria resistance pattern allowed them to continue treatment without vancomycin. The causes of inclusion errors or drop-outs are listed in Fig. 1, and the outcomes of the drop-out patients are listed in Table 5.

\section{Discussion}

In this multicenter, randomized controlled trial involving 48 patients with acute staphylococcal PJI treated with a DAIR procedure, the addition of rifampin to standard treatment with cloxacillin or vancomycin did not improve the cure rate. To our knowledge, this is the second randomized controlled trial to examine the effectiveness of adjuvant rifampin therapy in acute PJIs, and our findings are in contrast to previous findings. Zimmerli et al. published the first study back in 1998 [23]. It was a

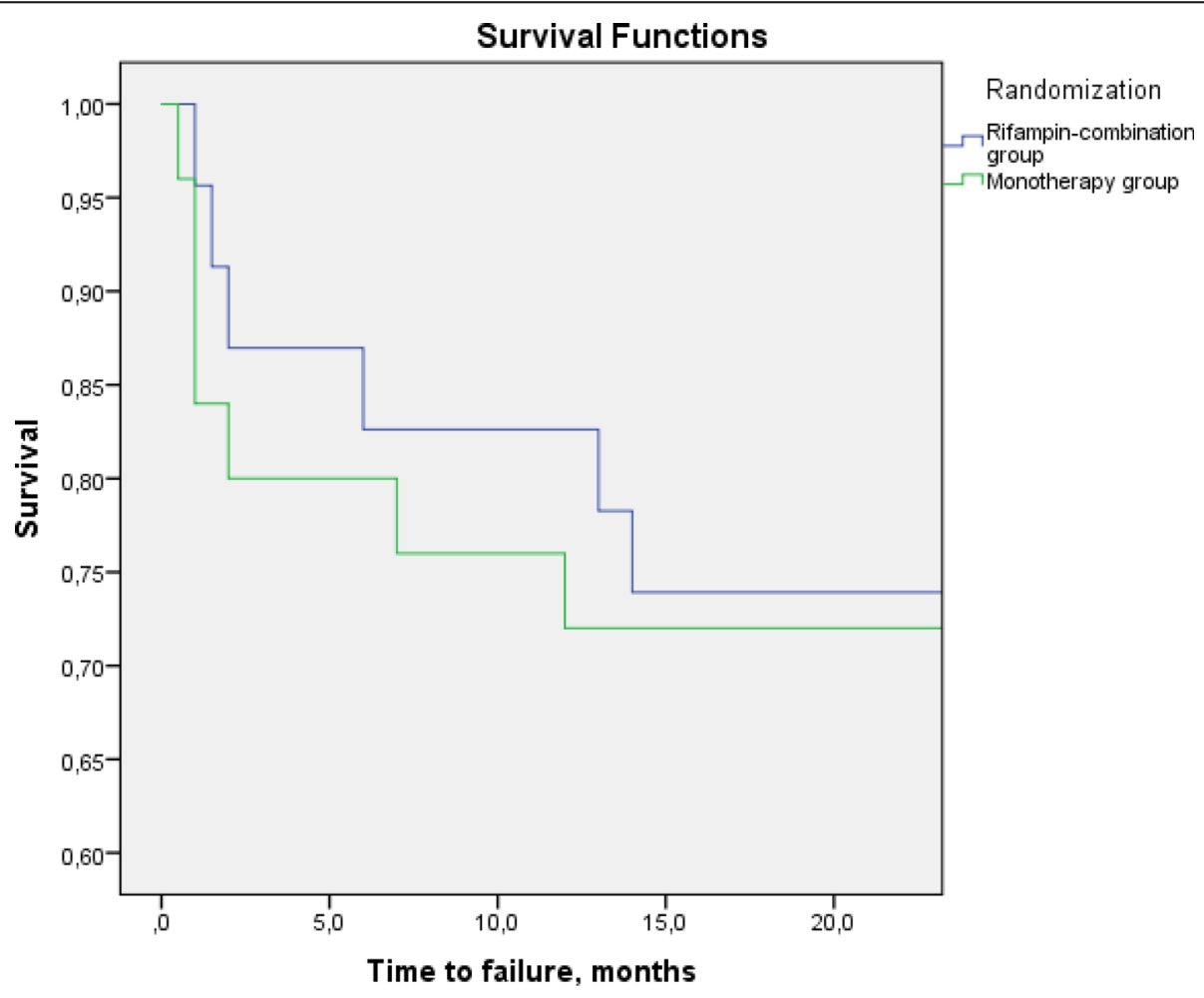

$\mathrm{CI}$ in the rifampin-combination group: $18-30$

$\mathrm{CI}$ in the monotherapy group: 18 - 31

Fig. 2 Kaplan Meier survival curve 
Table 3 Subanalyses according to type of staphylococci

\begin{tabular}{llll}
\hline a & & Success & Failure \\
\hline Rifampicin group & MSSA & $14 / 18$ & $4 / 18$ \\
\multirow{2}{*}{ Monotherapy group } & MRSE & $3 / 5$ & $2 / 5$ \\
& MSSA & $13 / 20$ & $7 / 20$ \\
Total & MRSE & $5 / 5$ & $0 / 5$ \\
& MSSA & $27 / 38$ & $11 / 38$ \\
& MRSE & $8 / 10$ & $2 / 10$ \\
\hline
\end{tabular}

${ }^{a}$ MSSA methicillin-susceptible S. aureus, MRSE methicillin-resistant S. epidermidis

single-center trial involving 24 patients, of which 15 were PJIs and 9 were infected osteosyntheses. This study was prematurely discontinued because all the failures occurred in the control group. It has been criticized for small numbers and limited statistical power, 33\% dropout rate in the rifampin group, as well as the choice of ciprofloxacin as monotherapy in the control group. Due to the concerns by using ciprofloxacin in monotherapy, we chose to add rifampin to the standard treatment at the time, which was cloxacillin or vancomycin in case of methicillin resistance.

Several retrospective observational studies and case series have been published in the last decades, evaluating different rifampin combinations. These studies were not controlled studies, and the success rates have never reached $100 \%$ as in the Zimmerli study, but their findings have although favored the use of rifampin [25, 27, 28]. There are difficulties in the interpretation of these studies, including considerable differences in baseline characteristics between treatment groups, surgical methods not described in detail, and varying MRSA rates. Barberán et al. found a success rate of $65 \%$ in staphylococcal PJIs treated with rifampin and levofloxacin following DAIR, seemingly a more common and expected result from these infections [29]. In a recently published study, a significant higher failure rate was found in rifampin combinations with linezolid, cotrimaxazole, and clindamycin. This is explained by the fact that rifampin reduces the serum concentration of

Table 4 Bacterial findings at rerevisions due to failure

\begin{tabular}{lll}
\hline Microbes & Rifampin group & Monotherapy group \\
\hline MSSA & 0 & 2 \\
MRSE & 2 & 1 \\
MRSE + Enterococcus faecalis & 0 & 1 \\
No growth & 2 & 3 \\
Not taken & 1 & 0 \\
Not revised & 1 & 0 \\
Total & 6 & 7 \\
\hline
\end{tabular}

these drugs and also for fusidic acid [30, 31]. Two review articles from 2008 and 2010, respectively, both conclude that the use of rifampin is based mostly on noncomparable in vitro and in vivo data and retrospective case reviews. Because of the biases of these, there are not sufficient data to support rifampin combination therapies $[32,33]$. However, it suggests that it could be effective in infections containing biofilm-producing agents, such as staphylococcal PJIs, but its use must be evaluated against the probability of drug interference and toxicity for each individual patient. A retrospective study from 2017 proved no advantage in treating streptococcal infections with an addition of rifampin [34]. These infections also form biofilm, and rifampin should theoretically improve the outcome. In vitro studies are inconsistent, but most have shown that the combination of vancomycin and rifampin promotes antagonism or indifference [35]. An in vivo case report showed a higher failure rate in PJIs when combining vancomycin and rifampin after debridement and retention of the prosthesis [29]. This is consistent with our results and the combination vancomycin-rifampin should be used cautiously. An RCT on staphylococcal bacteraemia proved no benefit from adding rifampin to the standard antibiotic therapy [36].

The preferred treatment in early PJI is DAIR. This option reduces morbidity, improves function, and is costeffective compared to 1 - and 2-stage revisions [11, 37]. It has been postulated that the biofilm has increased to such a degree that cure with DAIR is less achievable after 1 month [38]. Only patients with infections within 4 weeks after surgery, and acute hematogenous infections, were included in our study. The reported results following DAIR have been varying considerably, ranging from $21 \%$ to $100 \%$ [13, 19, 23, 27, 39]. There are many limitations when interpreting the literature, as several factors are varying and the surgical procedures are often poorly described. Both the definition of acute PJI, the number of procedures, the type and duration of antibiotics, and even the definition of success vary. Our results of approximately $75 \%$ success at 2 years of follow-up without suppressive antimicrobial therapy are comparable with recent literature $[40,41]$. Regarding the number of procedures, some advocates that repeated DAIR are effective [42]. On the other hand, some authors have found the need for additional DAIRs is associated with increased risk of failures [43]. In this present study, we chose to regard an additional DAIR procedure as a failure.

We found $S$. aureus to be more frequent than CoNS in this material, which is in contrast to most reports [12, 14, 30]. This may reflect the challenges of defining PJI. We used the Tsukayama definition, including patients with a short duration of symptoms shortly after the 
Table 5 Outcome of dropout patients*

\begin{tabular}{|c|c|c|c|}
\hline Initial therapy & Therapy after dropout & Cure & Failure \\
\hline \multirow[t]{2}{*}{ Cloxacillin and rifampin } & Cloxacillin & 4 & 1 \\
\hline & Cloxacillin and cotrimoxazol & 1 & \\
\hline \multirow[t]{2}{*}{ Vancomycin and rifampin } & Vancomycin and linezolid & 1 & \\
\hline & Rifampin and linezolid & 1 & \\
\hline \multirow[t]{4}{*}{ Cloxacillin } & Cloxacillin & 1 & \\
\hline & Fusidic acid and rifampin & & 1 \\
\hline & Ciprofloxacin and cloxacillin & 1 & \\
\hline & Clindamycin & 1 & \\
\hline \multirow[t]{3}{*}{ Vancomycin } & Linezolid and trimetoprim-sulfa & & 1 \\
\hline & Ciprofloxacin and cloxacillin & 1 & \\
\hline & Clindamycin & & 1 \\
\hline
\end{tabular}

*Two dropout-patients are not registred in the table due to withdrawal of consent and lost to follow-up

index surgery (30 days) [13]. Many definitions include PJI within the first 3 months after index surgery as early postoperative infections. This may explain the findings of more virulent bacteria (S. aureus) in our material.

PJI caused by methicillin-resistant S. aureus (MRSA) is reported to worsen the prognosis of DAIR, and a rifampin-containing antimicrobial regimen is often used. There were no MRSA infections in our cohort, reflecting the still very low prevalence of these infections in the Nordic countries. A prevelance $<1 \%$ is reported in Norway over the last 20 years [44].

The strengths of the trial are its randomized, multicenter design, which provides generalizable findings. It is also the largest RCT to date examining adjunctive rifampin in the treatment of staphylococcal PJI. There are several limitations to the study. First of all, the sample size is relatively small, but the 65 patients included doubles the numbers included in the previous trial. Recruitment rates were lower than expected at all participating sites, illustrating why conducting randomized clinical trials in clinical settings with few eligible patients is difficult. The study was stopped after enrolment of 99 patients, without knowledge of study outcome. A small sample size increases the risk of type II error and then often showing high intervention effects. In this study, there was an independent reason for stopping the trial, which may lower the risk of bias. One may argue that statistical power is a pre-study tool, and because our study ended due to slow inclusion rates, the actual results may be interpreted as they are, based on the $95 \%$ CIs [45]. The 95\% CIs around our estimates of the difference between the groups were quite small. Secondly, gentamicin collagen sponges were placed in the wound before closure. This adds an additional antimicrobial agent to the equation and was performed according to the guidelines at the time of initiating the study. However, all patients received these sponges, and it is difficult to see how it could have affected the final outcome. Third, the type of antibiotics combined with rifampin seems to affect the outcome. Our results may therefore not be comparable with other rifampin combinations.

In conclusion, our findings indicate that adding rifampin to cloxacillin or vancomycin treatment in patients with acute staphylococcal PJIs does not affect the cure rate of a DAIR procedure. Given the substantial risk of drug interactions and the risk of increasing bacterial resistance, we find that adjunctive rifampin provides no benefit over standard treatment. The results must however be cautiously interpreted due to a low number of patients, but the study still adds important knowledge to defining the benefit of rifampin.

\section{Abbreviations}

PJI: Prosthetic joint infection; DAIR: Debridement, antibiotics, and implant retention; MRSE: Methicillin-resistant Staphylococcus epidermidis;

CoNS: Coagulase-negative Staphylococci; CRP: C-reactive protein; ESR: Erythrocyte sedimentation rate; MRSA: Methicillin-resistant

Staphylococcus aureus; MSSA: Methicillin-susceptible Staphylococcus aureus;

$\mathrm{Cl}$ : Confidence interval

\section{Acknowledgements}

We are grateful to Dr. Bent von der Lippe of the Department of Infectious Diseases, Oslo University Hospital, for his contribution in initiating the study.

\section{Authors' contributions}

All the authors have made a significant contribution in collecting the data and in the development of the study and finishing the manuscript. The authors read and approved the final manuscript.

\section{Funding}

The study was funded by the participating hospitals partly as part of our routine work and partly by internal funding to supplementary outpatient clinic hours and X-rays. It was also made possible with a contribution from Sophie Mindes Stiftelse, a non-profit organization, giving grants to orthopedic research.

\section{Availability of data and materials}

The datasets used and/or analyzed during the current study are available from the corresponding author on reasonable request. 


\section{Ethics approval and consent to participate}

The Regional Ethics Committee and the Norwegian Medicines Agency have approved this study. All patients participating approved by written consent.

\section{Consent for publication}

Not applicable

\section{Competing interests}

The authors have no conflict of interest

\section{Author details}

'Division of Orthopaedic Surgery, Oslo University Hospital, Oslo, Norway. ${ }^{2}$ Department of Orthopaedic Surgery, Betanien Hospital, Skien, Norway. ${ }^{3}$ Department of Orthopaedic Surgery, Martina Hansen Hospital, Bærum Norway. ${ }^{4}$ Department of Orthopaedic Surgery, Vestre Viken HF, Drammen, Norway. ${ }^{5}$ Department of Orthopaedic Surgery, Bærum Hospital, Bærum, Norway. ${ }^{6}$ Department of Orthopaedic Surgery, Sykehuset Innlandet HF, Lillehammer, Norway. ${ }^{7}$ Department of Orthopaedic Surgery, Sykehuset Innlandet HF, Elverum, Norway. ${ }^{8}$ Department of Orthopaedic Surgery, St. Olavs Hospital, Trondheim, Norway.

Received: 14 February 2020 Accepted: 10 August 2020

\section{Published online: 28 August 2020}

\section{References}

1. Kurtz SM, et al. Future clinical and economic impact of revision total hip and knee arthroplasty. J Bone Joint Surg Am. 2007;89(Suppl 3):144-51.

2. Harris $\mathrm{WH}$, Sledge CB. Total hip and total knee replacement (2). N Engl J Med. 1990;323(12):801-7.

3. Darouiche RO. Treatment of infections associated with surgical implants. $N$ Engl J Med. 2004;350(14):1422-9.

4. Bassetti $M$, et al. Linezolid in the treatment of gram-positive prosthetic joint infections. J Antimicrob Chemother. 2005:55(3):387-90.

5. Lentino JR. Prosthetic joint infections: bane of orthopedists, challenge for infectious disease specialists. Clin Infect Dis. 2003;36(9):1157-61.

6. Zmistowski $B$, et al. Periprosthetic joint infection increases the risk of oneyear mortality. J Bone Joint Surg Am. 2013;95(24):2177-84.

7. Kurtz SM, et al. Economic burden of periprosthetic joint infection in the United States. J Arthroplast. 2012;27(8 Suppl):61-5 e1.

8. Dale $\mathrm{H}$, et al. Increasing risk of prosthetic joint infection after total hip arthroplasty. Acta Orthop. 2012;83(5):449-58

9. Kurtz SM, et al. Infection burden for hip and knee arthroplasty in the United States. J Arthroplast. 2008;23(7):984-91.

10. Trampuz A, Zimmerli W. New strategies for the treatment of infections associated with prosthetic joints. Curr Opin Investig Drugs. 2005;6(2):185-90.

11. Fisman DN, et al. Clinical effectiveness and cost-effectiveness of 2 management strategies for infected total hip arthroplasty in the elderly. Clin Infect Dis. 2001:32(3):419-30.

12. Moran E, et al. Guiding empirical antibiotic therapy in orthopaedics: the microbiology of prosthetic joint infection managed by debridement, irrigation and prosthesis retention. J Inf Secur. 2007;55(1):1-7.

13. Tsukayama DT, Estrada R, Gustilo RB. Infection after total hip arthroplasty. A study of the treatment of one hundred and six infections. J Bone Joint Surg Am. 1996;78(4):512-23

14. Stefansdottir A, et al. Microbiology of the infected knee arthroplasty: report from the Swedish knee arthroplasty register on 426 surgically revised cases. Scand J Infect Dis. 2009:41(11-12):831-40.

15. Gristina $A G$, et al. Adhesive colonization of biomaterials and antibiotic resistance. Biomaterials. 1987:8(6):423-6.

16. Zimmerli W, Trampuz A, Ochsner PE. Prosthetic-joint infections. N Engl J Med. 2004:351(16):1645-54.

17. Stewart PS, Costerton JW. Antibiotic resistance of bacteria in biofilms. Lancet. 2001;358(9276):135-8.

18. Lister JL, Horswill AR. Staphylococcus aureus biofilms: recent developments in biofilm dispersal. Front Cell Infect Microbiol. 2014;4:178.

19. Westberg M, Grogaard B, Snorrason F. Early prosthetic joint infections treated with debridement and implant retention: 38 primary hip arthroplasties prospectively recorded and followed for median 4 years. Acta Orthop. 2012;83(3):227-32.
20. Van Kleunen JP, et al. Irrigation and debridement and prosthesis retention for treating acute periprosthetic infections. Clin Orthop Relat Res. 2010; 468(8):2024-8.

21. Fazly Bazzaz BS, et al. Antibacterial efficacy of rifampin loaded solid lipid nanoparticles against Staphylococcus epidermidis biofilm. Microb Pathog. 2016:93:137-44.

22. Riedel DJ, Weekes E, Forrest GN. Addition of rifampin to standard therapy for treatment of native valve infective endocarditis caused by Staphylococcus aureus. Antimicrob Agents Chemother. 2008;52(7): 2463-7.

23. Zimmerli $\mathbf{W}$, et al. Role of rifampin for treatment of orthopedic implant-related staphylococcal infections: a randomized controlled trial. Foreign-body infection (FBI) study group. JAMA. 1998;279(19): 1537-41.

24. El Helou OC, et al. Efficacy and safety of rifampin containing regimen for staphylococcal prosthetic joint infections treated with debridement and retention. Eur J Clin Microbiol Infect Dis. 2010;29(8):961-7.

25. Senneville $E$, et al. Outcome and predictors of treatment failure in total hip/ knee prosthetic joint infections due to Staphylococcus aureus. Clin Infect Dis. 2011:53(4):334-40.

26. Berdal JE, et al. Use of rifampicin and ciprofloxacin combination therapy after surgical debridement in the treatment of early manifestation prosthetic joint infections. Clin Microbiol Infect. 2005; 11(10):843-5.

27. Lora-Tamayo J, et al. A large multicenter study of methicillin-susceptible and methicillin-resistant Staphylococcus aureus prosthetic joint infections managed with implant retention. Clin Infect Dis. 2013;56(2):182-94.

28. Holmberg A, et al. 75\% success rate after open debridement, exchange of tibial insert, and antibiotics in knee prosthetic joint infections. Acta Orthop. 2015:86(4):457-62

29. Barberan J, et al. Conservative treatment of staphylococcal prosthetic joint infections in elderly patients. Am J Med. 2006;119(11):993 e7-10.

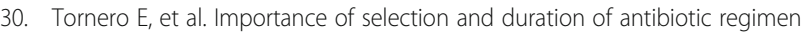
in prosthetic joint infections treated with debridement and implant retention. J Antimicrob Chemother. 2016;71(5):1395-401.

31. Pushkin $\mathrm{R}$, et al. A randomized study evaluating oral fusidic acid (CEM-102) in combination with oral rifampin compared with standard-of-care antibiotics for treatment of prosthetic joint infections: a newly identified drug-drug interaction. Clin Infect Dis. 2016;63(12):1599-604

32. Forrest GN, Tamura K. Rifampin combination therapy for nonmycobacterial infections. Clin Microbiol Rev. 2010;23(1):14-34.

33. Perlroth J, et al. Adjunctive use of rifampin for the treatment of Staphylococcus aureus infections: a systematic review of the literature. Arch Intern Med. 2008;168(8):805-19.

34. Akgun $D$, et al. High failure rates in treatment of streptococcal periprosthetic joint infection: results from a seven-year retrospective cohort study. Bone Joint J. 2017:99-B(5):653-9.

35. Shelburne SA, et al. In vitro killing of community-associated methicillinresistant Staphylococcus aureus with drug combinations. Antimicrob Agents Chemother. 2004;48(10):4016-9.

36. Thwaites GE, et al. Adjunctive rifampicin for Staphylococcus aureus bacteraemia (ARREST): a multicentre, randomised, double-blind, placebocontrolled trial. Lancet. 2018:391(10121):668-78.

37. Grammatopoulos G, et al. Functional outcome of debridement, antibiotics and implant retention in periprosthetic joint infection involving the hip: a case-control study. Bone Joint J. 2017;99-B(5):614-22.

38. Bernard $L$, et al. Trends in the treatment of orthopaedic prosthetic infections. J Antimicrob Chemother. 2004;53(2):127-9.

39. Crockarell JR, et al. Treatment of infection with debridement and retention of the components following hip arthroplasty. J Bone Joint Surg Am. 1998; 80(9):1306-13.

40. Tsang SJ, et al. Outcomes following debridement, antibiotics and implant retention in the management of periprosthetic infections of the hip: a review of cohort studies. Bone Joint J. 2017:99-B(11):1458-66.

41. Kuiper JW, et al. Prosthetic joint-associated infections treated with DAIR (debridement, antibiotics, irrigation, and retention): analysis of risk factors and local antibiotic carriers in 91 patients. Acta Orthop. 2013; 84(4):380-6.

42. Mont MA, et al. Multiple irrigation, debridement, and retention of components in infected total knee arthroplasty. J Arthroplast. 1997:12(4): 426-33. 
43. Moojen DJ, et al. Similar success rates for single and multiple debridement surgery for acute hip arthroplasty infection. Acta Orthop. 2014;85(4):383-8.

44. Moxnes JF, et al. Methicillin-resistant Staphylococcus aureus (MRSA) is increasing in Norway: a time series analysis of reported MRSA and methicillin-sensitive S. aureus cases, 1997-2010. PLoS One. 2013;8(8):e70499.

45. Goodman SN, Berlin JA. The use of predicted confidence intervals when planning experiments and the misuse of power when interpreting results. Ann Intern Med. 1994;121(3):200-6.

\section{Publisher's Note}

Springer Nature remains neutral with regard to jurisdictional claims in published maps and institutional affiliations.

Ready to submit your research? Choose BMC and benefit from:

- fast, convenient online submission

- thorough peer review by experienced researchers in your field

- rapid publication on acceptance

- support for research data, including large and complex data types

- gold Open Access which fosters wider collaboration and increased citations

- maximum visibility for your research: over $100 \mathrm{M}$ website views per year

At $B M C$, research is always in progress.

Learn more biomedcentral.com/submissions 